\title{
Comparative cytology, physiology and transcriptomics of Burkholderia insecticola in symbiosis with the bean bug Riptortus pedestris and in culture
}

\author{
Tsubasa Ohbayashi ${ }^{1,2} \cdot$ Ryo Futahashi $\mathbb{D}^{3} \cdot$ Mia Terashima $a^{2,4} \cdot$ Quentin Barrière ${ }^{1} \cdot$ Florian Lamouche $^{1}$. \\ Kazutaka Takeshita ${ }^{2,8} \cdot$ Xian-Ying Meng $^{3} \cdot$ Yasuo Mitani $^{3} \cdot$ Teruo Sone $^{2} \cdot$ Shuji Shigenobu $^{5} \cdot$ Takema Fukatsu $\mathbb{D}^{3} \cdot$ \\ Peter Mergaert (D) $^{1} \cdot$ Yoshitomo Kikuchi iD $^{2,6,7}$
}

Received: 13 April 2018 / Revised: 4 December 2018 / Accepted: 19 January 2019 / Published online: 11 February 2019

(c) International Society for Microbial Ecology 2019

\begin{abstract}
In the symbiosis of the bean bug Riptortus pedestris with Burkholderia insecticola, the bacteria occupy an exclusive niche in the insect midgut and favor insect development and reproduction. In order to understand how the symbiotic bacteria stably colonize the midgut crypts and which services they provide to the host, we compared the cytology, physiology, and transcriptomics of free-living and midgut-colonizing $B$. insecticola. The analyses revealed that midgut-colonizing bacteria were smaller in size and had lower DNA content, they had increased stress sensitivity, lost motility, and an altered cell surface. Transcriptomics revealed what kinds of nutrients are provided by the bean bug to the Burkholderia symbiont. Transporters and metabolic pathways of diverse sugars such as rhamnose and ribose, and sulfur compounds like sulfate and taurine were upregulated in the midgut-colonizing symbionts. Moreover, pathways enabling the assimilation of insect nitrogen wastes, i.e. allantoin and urea, were also upregulated. The data further suggested that the midgut-colonizing symbionts produced all essential amino acids and B vitamins, some of which are scarce in the soybean food of the host insect. Together, these findings suggest that the Burkholderia symbiont is fed with specific nutrients and also recycles host metabolic wastes in the insect gut, and in return, the bacterial symbiont provides the host with essential nutrients limited in the insect food, contributing to the rapid growth and enhanced reproduction of the bean bug host.
\end{abstract}

\section{Introduction}

Mutualistic bacteria cooperate with their host by providing services, and at the same time, they have to be able to stably

Supplementary information The online version of this article (https:// doi.org/10.1038/s41396-019-0361-8) contains supplementary material, which is available to authorized users.

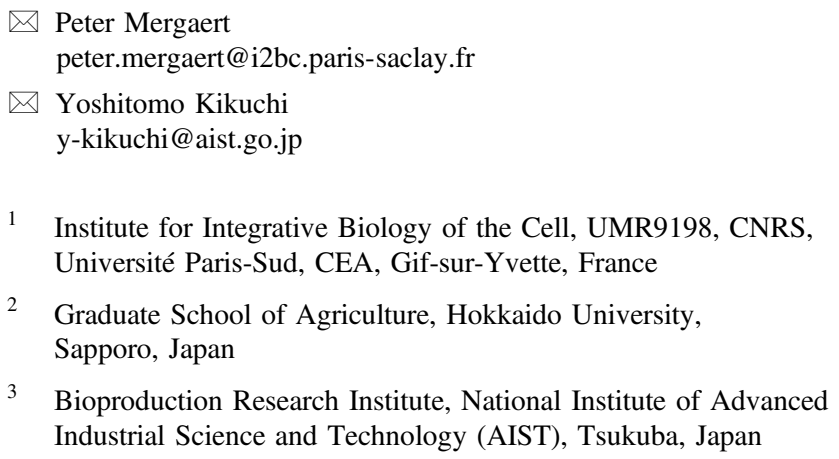

3 Bioproduction Research Institute, National Institute of Advanced Industrial Science and Technology (AIST), Tsukuba, Japan

colonize the internal environment of the eukaryotic host and to overcome host control mechanisms. To accomplish this, mutualists often alter their morphology, physiology, cytology, and sometimes even their genomic composition [1, 2]. Insects, that feed on nutritionaly unbalanced or undigestable diets, commonly possess mutualistic bacteria in specialized organs, such as gut crypts and bacteriomes [3-5], wherein symbionts play pivotal metabolic roles, including provision

4 Institute of Low Temperature Science, Hokkaido University, Sapporo, Japan

5 NIBB Core Research Facilities, National Institute for Basic Biology, Okazaki, Japan

6 Computational Bio Big Data Open Innovation Laboratory (CBBDOIL), AIST, Sapporo, Japan

7 Bioproduction Research Institute, AIST, Sapporo, Japan

8 Present address: Faculty of Bioresource Sciences, Akita Prefectural University, Akita, Japan 
of essential nutrients [6, 7], digestion of plant cell-wall components [8-10], and reutilization of metabolic wastes of the host $[6,11]$. In addition to such nutritional contributions, microbial symbionts are also involved in defense against natural enemies [12, 13], sex determination [14], and detoxification of phytotoxins and pesticides [15]. Most of the insect symbionts are vertically transmitted and highly adapted to their host's internal environment [5, 16]. As a result of, in the evolutionary scale, long-term exclusive residence within the insect hosts, the symbionts tend to lose genes that are not required for life in these unchanging conditions, including genes for flagella motility, cell-wall biosynthesis, transporters, and secretion systems [7, 17, 18]. Such reduced genomes of symbionts are thought to contribute to rapid reproduction and efficient service-provision inside insect hosts.

The bean bug Riptortus pedestris (Hemiptera: Alydidae) is a notorious pest of leguminous crops and is broadly distributed in Southeast Asia [19-22]. The insect interacts intimately with a specific symbiont called Burkholderia insecticola [23], a dense population of which is harbored in crypts in the posterior midgut region, called M4 (midgut $4^{\text {th }}$ section) [24]. In comparison with symbiotic insects, aposymbiotic insects have a prolonged developmental period, smaller body size and reduced egg numbers [25-27], indicating a pivotal metabolic role of the Burkholderia symbiont in the bean bug host. Exceptional among insects, $R$. pedestris and allied species do not vertically transmit their symbionts but instead acquire the specific Burkholderia symbiont from the soil every generation [25, 28, 29]. In the bean bug, the symbiont colonization starts and fully establishes within the $2^{\text {nd }}$ instar stage [26, 30]. Since the Burkholderia symbiont can be cultivated and genetically manipulated, the Riptortus-Burkholderia symbiotic system is an attractive model for clarifying the genetic and molecular basis of insect-microbe symbiotic associations [24, 31]. Previous studies have revealed that the Burkholderia symbiont requires its flagellar motility to reach the symbiotic organ [32, 33], and a specific lipopolysaccharide (LPS) structure and other envelope molecules as well as polyhydroxyalkanoate (PHA) metabolism for a full and lasting colonization of the symbiotic organ [34-38].

In $R$. pedestris, the M4 region as well as a distinct, adjacent midgut region called the M4 bulb (M4B), produce specifically a diverse set of digestive enzymes and antimicrobial peptides, including a large family of cysteine-rich peptides which we call the crypt-specific cysteine-rich peptides (CCRs) [36, 39-42]. This suggests that the Burkholderia symbiont is exposed to various stresses in the symbiotic organ. It remains largely unknown how the Burkholderia symbiont alters its physiology to cope with the specific conditions of the gut environment of the bean bug host. In addition, the metabolic basis of the benefits that the insect host obtains from the bacterial symbionts is still unclear.

To understand the genetic and physiological features and the metabolic activity of $B$. insecticola in the host internal environment, we compared here the cell morphology and cytology by microscopic observations, the bacterial physiology by stress response tests, and transcriptomics by RNA-sequencing (RNA-seq) between the free-living Burkholderia symbiont cultured in liquid media (in vitro) and symbiotic Burkholderia colonizing the midgut crypts (in vivo).

\section{Materials and methods}

\section{Insect and bacterial strains}

The bean bug was maintained in the laboratory as described previously [32] using soybeans as food source and distilled water containing $0.05 \%$ ascorbic acid as drinking water. $B$. insecticola strain RPE75 [25], a spontaneous rifampicinresistant mutant $\left(\mathrm{Rf}^{\mathrm{r}}\right)$ of the wild-type strain RPE64 [23], and its GFP (green fluorescent protein) labeled derivative RPE225 [26] were used in this study.

\section{Preparation of in vitro symbiont cells}

The Burkholderia symbiont was grown in YG and/or MM medium (Supplementary Table S1) containing rifampicin $(10 \mu \mathrm{g} / \mathrm{ml})$ on a gyratory shaker $(150 \mathrm{rpm})$ at $27^{\circ} \mathrm{C}$, and cultured cells were centrifuged and washed twice with phosphate-buffered saline (PBS) before analysis (Supplementary Table S1).

\section{Preparation of in vivo symbiont cells}

The oral administration of the symbiont to the insect and its dissection were performed as described previously [32]. Three days old (second instar) insects were infected by adding the symbiont to their drinking water $\left(10^{7} \mathrm{cfu} / \mathrm{mL}\right)$. Symbiotic organs were collected by dissection and the pooled midgut crypts of 10-30 insects were homogenized by a pestle, centrifuged, washed twice with PBS, and then subjected for further analyses. The harvests were performed at the $3^{\text {rd }}$ or $5^{\text {th }}$ instar $(5$ or 12 days after infection, respectively), depending on the experiments (see Supplementary Table S1).

\section{Stress-sensitivity assays}

Suspensions of the in vivo and in vitro symbiont cells were exposed to 13 different antimicrobial chemicals (Supplementary Table S2) for $1 \mathrm{~h}$ at $27^{\circ} \mathrm{C}$. These cells were diluted, 
inoculated on YG-Rf plates, and incubated at $27^{\circ} \mathrm{C}$ for 2 days. Bacterial growth was determined by $\mathrm{CFU}$ counting and data are presented as relative CFU numbers. Further details of the method are provided in Supplementary Information.

\section{RNA-sequencing analysis}

Total RNA was extracted from triplicate samples from in vivo symbiont cells and $3 \mathrm{~h}$ - (mean $\left.\mathrm{OD}_{600}=0.27\right), 8 \mathrm{~h}$ $\left(\right.$ mean $\left.\mathrm{OD}_{600}=1.87\right)$, and $16 \mathrm{~h}-\left(\right.$ mean $\left.\mathrm{OD}_{600}=6.38\right)$ cultured in vitro symbiont cells by using trizol (Thermo Scientific, Wilmington, NC, USA), the RNeasy mini kit (Qiagen Inc., Valencia, CA, USA), and the TURBO DNAfree kit (Thermo Scientific, Wilmington, NC, USA). The mRNA was further enriched by the Eukaryote Human/ Mouse/Rat and/or the Bacteria Ribo-Zero kits (Illumina, Inc., San Diego, CA, USA). The cDNA libraries were constructed from approximately $1 \mu \mathrm{g}$ of total RNA by use of the Scriptseq v2 RNA-seq library kit (Illumina, Inc., San Diego, CA, USA). In total, 12 cDNA libraries were constructed, and sequenced by Nextseq (ver. NS500446, Illumina, Inc., San Diego, CA, USA). The obtained RNA-seq data were analyzed by the bcl2fastq software (ver. 22.18.12, Illumina, Inc., San Diego, CA, USA), Cutadapt (ver. 1.15 [43]) for adapter trimming, FastQC (ver. 0.11.5 [http://www.bioinformatics.babraham.ac.uk/projects/fastqc/ ]) for quality control, and BWA (ver. 0.6.2-r126 [44]) for mapping on the symbiont genome (GCA_00402035.1 [45]). Comparisons of gene expression levels were performed by Wald-tests in the DESeq2 program (version 1.12.4 [46]). Genes specifically upregulated and downregulated in vivo were defined as genes whose false discovery rate (FDR) $q$ values were $<0.01$ and expression fold changes were $>2$ or $<-0.5$ when their expressions were compared to $3 \mathrm{~h}-, 8 \mathrm{~h}$-, and $16 \mathrm{~h}$-cultured in vitro cells. Principal component analysis and heatmap presentations were performed by the FactoMineR (ver. 1.33 [47]) and the gplots package (http:// cran.r-project.org/web/packages/gplots/index.html), respectively.

\section{Nucleotide sequence accession number}

The RNA-seq nucleotide sequences reported in this study were deposited in the DDBJ/Genbank/EBI databases under the accession numbers: PRJDB6699, DRA007366, and SAMD00140959-SAMD00140970 (Supplementary Table S4).

\section{Other methods}

Methods for fluorescence microscopy, flow cytometry, electron microscopy, motility test, quantitative reverse- transcription PCR (qRT-PCR), colony PCR and statistical analyses are described in Supplementary Information.

\section{Results}

\section{Morphological and cytological alterations of in vivo Burkholderia symbionts}

Observed by light microscopy, in vitro symbiont cells were found to be large rods, as expected for a species of the genus Burkholderia. In contrast, in vivo symbiont cells appeared as small rods, close to cocci (Fig. 1a-d). The results of flow cytometry analysis by the light scatter corresponded to these microscopy observations and revealed a morphological alteration in the in vivo cells (Fig. 1e). The DAPI staining showed that the in vivo cells had a lower DNA content than the in vitro cells (Fig. 1f). The smaller size of the in vivo symbiont was furthermore obvious by their lower fluorescence intensity after staining with the cell membrane-specific, styryl-based dye FM4-64, (Fig. 1c, h). Together, these data imply that the cell size, DNA content and cell cycle of the Burkholderia symbiont undergo a marked shift during the colonization of the symbiotic organ. It may be relevant that in vitro, Burkholderia also become small with low DNA content when entering stationary phase or when grown in a minimal medium (see Supplementary Information; Supplementary Fig. S1). Nile red staining demonstrated that PHA (a bacterial carbon storage compound) accumulated in in vivo symbiont cells (Fig. 1b), although the flow cytometry analysis of a large number of cells showed that the PHA accumulation is in the total bacterial population not very different between the in vivo and in vitro bacteria (Fig. 1g).

The observation of in vitro and in vivo cells by TEM corroborated the features of cell shape and PHA accumulation observed by light microscopy and flow cytometry (Fig. 1j, k). Interestingly, frequent membrane blebs in 5 out of 12 cells were found on the surface of in vivo symbiont cells while they were not observed in in vitro cells (Fig. 1k; Supplementary Fig. S2).

The Burkholderia symbiont needs flagellar motility to reach and colonize the midgut crypts [32, 48]. However, testing the motility of in vivo and in vitro symbionts revealed that in vivo cells showed little motility at $16 \mathrm{~h}$ after incubation (Fig. 11) but that their flagellar mortility was recovered after $24 \mathrm{~h}$ incubation (Supplementary Fig. S3). This suggests that the symbiont loses its motility after having colonized M4 but that this process is reversible and motility is reactivated when the bacteria are released from the symbiotic organ. Taken together, these results demonstrate that the cellular characteristics of the Burkholderia are altered in the midgut environment. 
a

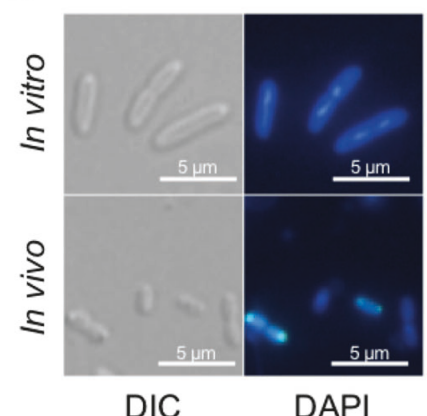

b

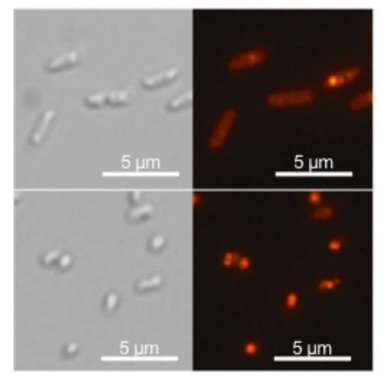

DIC c

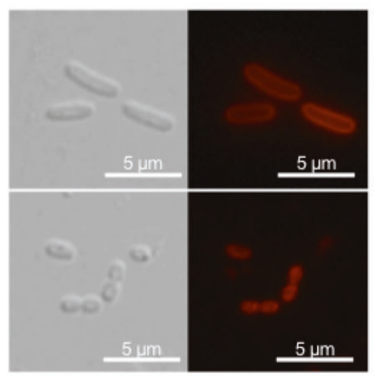

DIC d

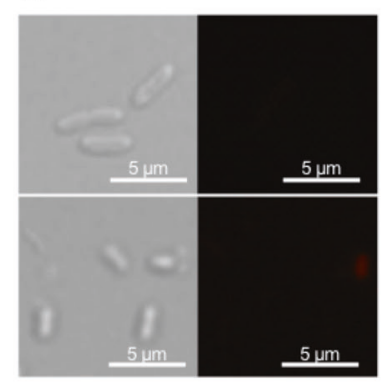

DIC
$\mathrm{Pl}$

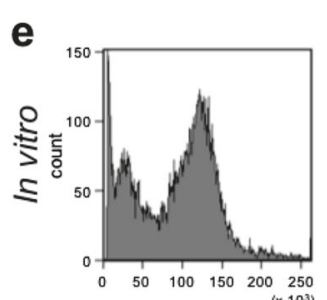

f

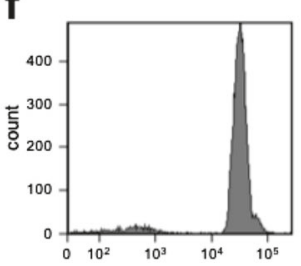

g

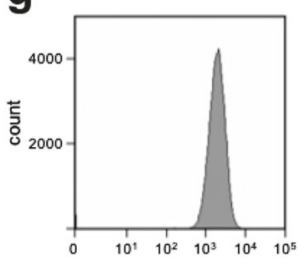

h

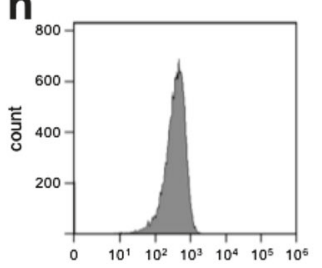

i
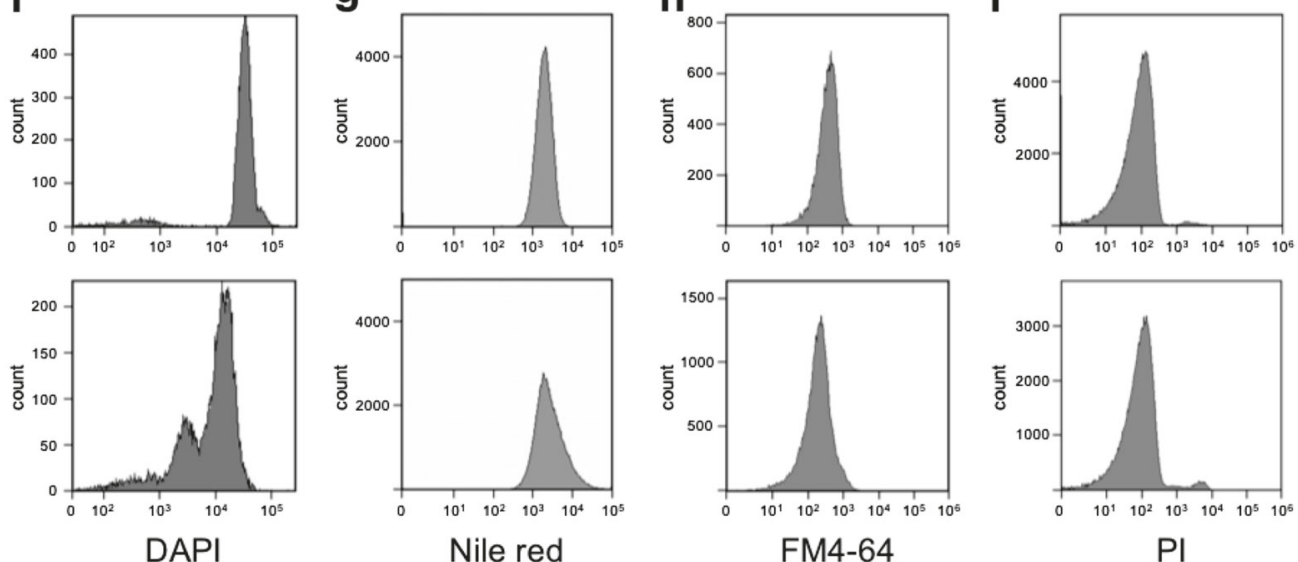

FM4-64

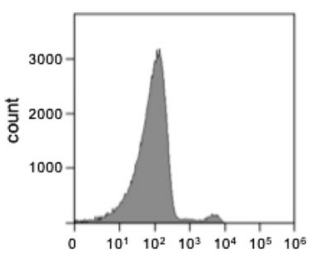

$\mathrm{Pl}$

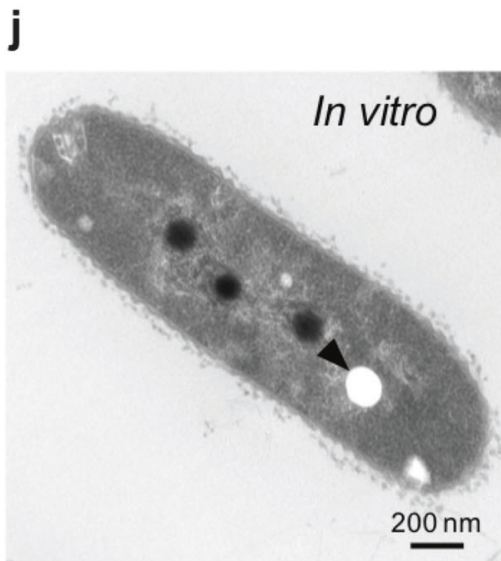

k
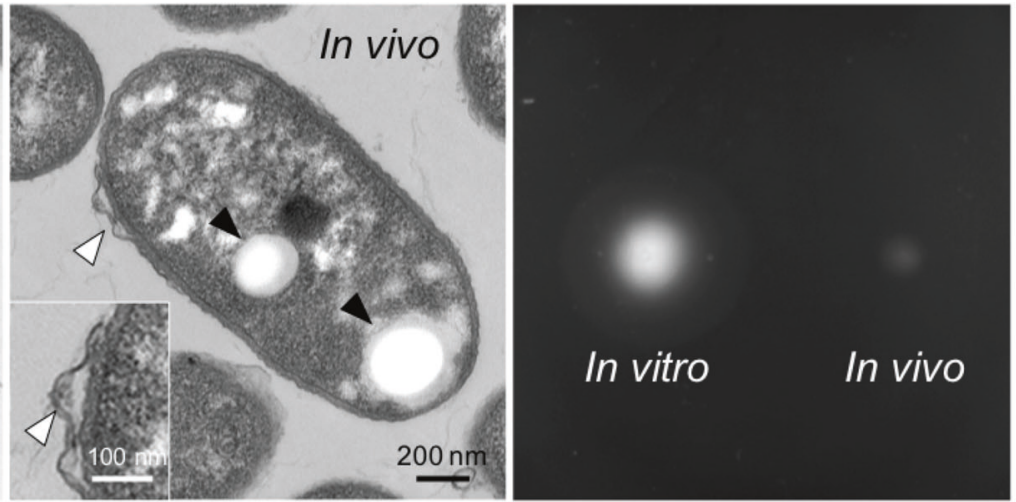

Fig. 1 Bacterial cell morphology of in vivo and in vitro Burkholderia symbiont cells. a-d Differential interference contrast (left) and fluorescence microscopy (right) images of in vitro and in vivo bacteria stained with DAPI a, Nile red b, FM4-64 c, and PI d. e-i Flow cytometry analysis of in vivo and in vitro symbiont cells measuring cell size by light scatter e, DNA content by DAPI staining f, PHA

\section{in vivo Burkholderia symbionts are more susceptible to various environmental stresses}

When symbiont cells were exposed to various antimicrobial compounds for a short time $(1 \mathrm{~h})$, in vivo symbionts were found to be more susceptible to accumulation by Nile red staining $\mathbf{g}$, and membrane area by FM4-64 staining $\mathbf{h}$, membrane permeability by PI staining $\mathbf{i}$. j, $\mathbf{k}$ Transmission electron microscopy images of an in vitro $\mathbf{j}$ and an in vivo $\mathbf{k}$ symbiont cell. Filled arrows indicate PHA granules and open arrows shows a membrane bleb. I Motility test of in vitro (left) and in vivo (right) cells (see also Supplementary Fig. S3)

membrane-disrupting agents, including SDS, proteinase $\mathrm{K}$, ethanol, and cationic antimicrobial peptides (AMPs) (polymyxin B and LL-37), reactive oxygen (hydrogen peroxide), salt $(\mathrm{NaCl})$, and high osmolarity (glucose) (Fig. 2; Supplementary Table S3). No obvious difference was detected between in vivo and in vitro cells in the 
Fig. 2 Comparison of stress sensitivities between in vivo and in vitro bacteria. The in vivo and in vitro Burkholderia symbiont cells were exposed to a SDS, b tween 20, c protease K, d LL-37, e polymyxin $\mathrm{B}$, $\mathbf{f}$ riptocin, g CCR0008, h CCR0179, i CCR0480, $\mathbf{j}$ hydrogen peroxide, $\mathbf{k}$ sodium chloride, $\mathbf{l}$ glucose, and $\mathbf{m}$ ethanol at the indicated concentrations. The relative growth $(\%)$ was normalized by setting untreated bacterial growth to $100 \%$. Mean $\pm \mathrm{SD}$ $(n=3)$ is shown in in vitro (solid bar) and in vivo cells (open bar). Statistically significant differences between in vivo and in vitro symbiont cells were analyzed by Student's $t$-test with Bonferroni correction: $* P<0.05$. CFU data of the stress tests are shown in

Supplementary Table S3
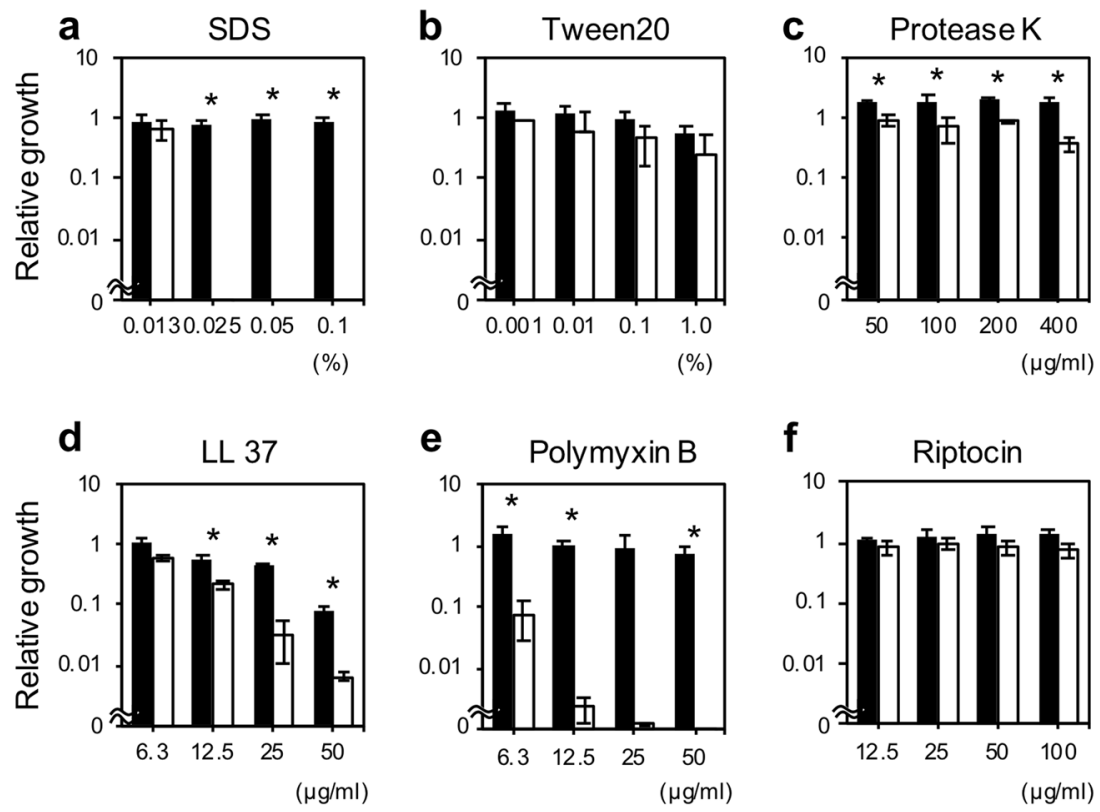

f
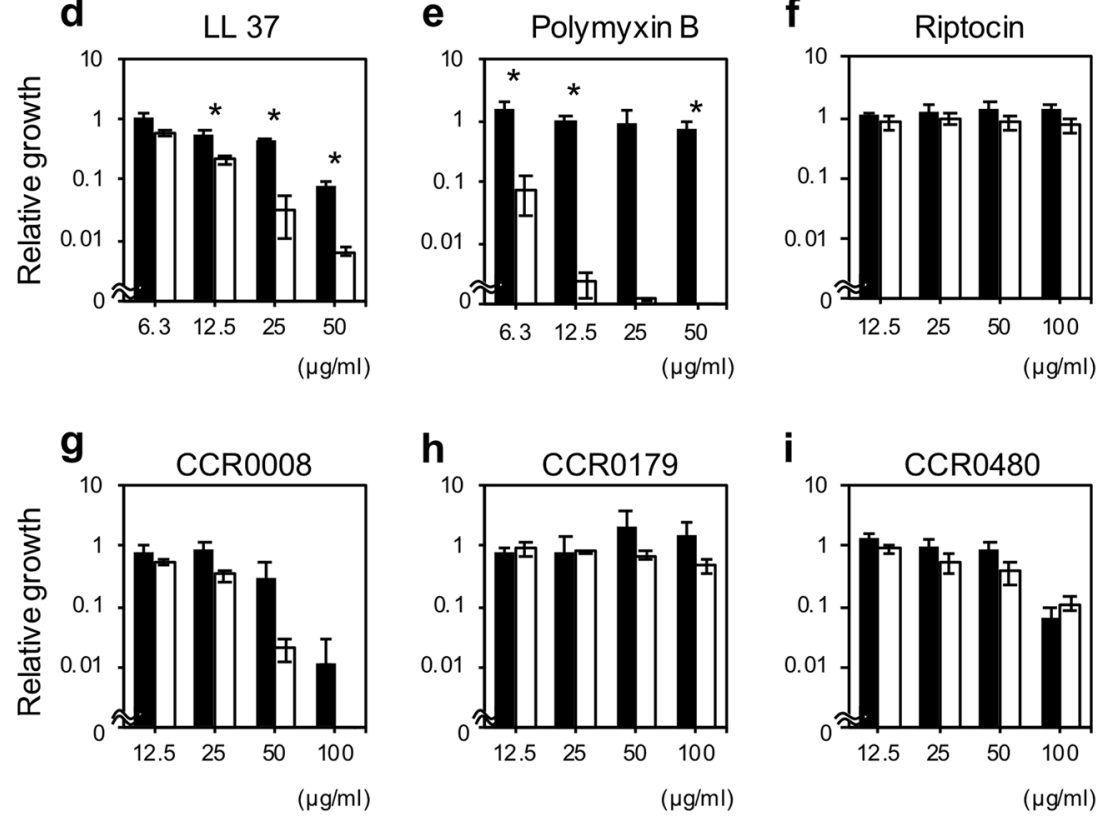

i
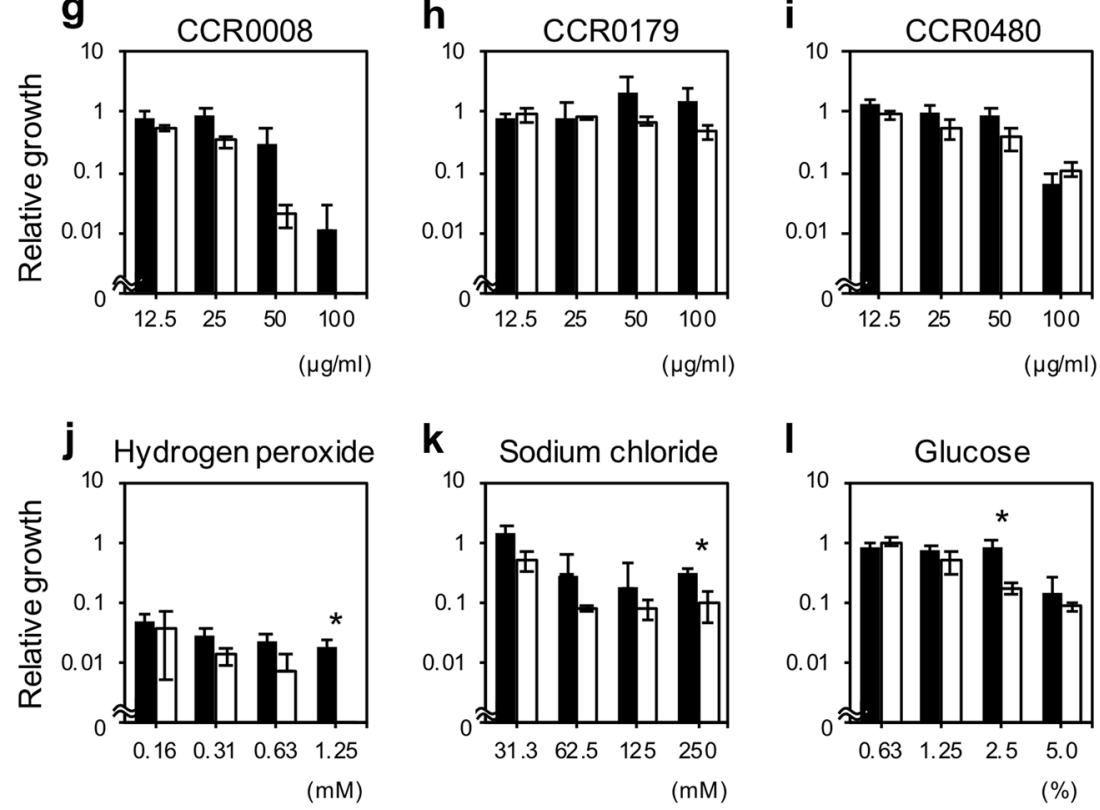

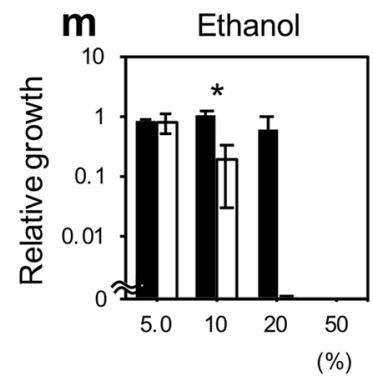

In vitro symbiont

In vivo symbiont

presence of the non-ionic detergent Tween 20, and three cationic symbiotic AMPs (CCR0008, CCR0179, and CCR0480) (Fig. 2; Supplementary Table S3). The higher sensitivity to some of the AMPs and detergents, which are both stresses that target the cell membrane, strongly suggests cell membrane alterations in the in vivo symbionts, which is in agreement with the TEM observations (Fig. 1k; Supplementary Fig. S2). 
RNA-sequencing of in vivo and in vitro Burkholderia symbionts

For a transcriptome comparison of the two Burkholderia cell-types, RNA-seq libraries were constructed from in vivo cells and $3 \mathrm{~h}-, 8 \mathrm{~h}-$, and $16 \mathrm{~h}$-cultured in vitro cells in tripricate (Supplementary Table S4). These in vitro cells corresponded to lag-phase, log-phase, and stationary-phase growing cells, respectively. More than 15,000,000 mapped reads were obtained from each in vitro sample, while over $5,800,000$ mapped reads were obtained from each in vivo sample (Supplementary Table S4). In order to verify the RNA-seq data, qRT-PCR was performed on 8 randomly selected genes (Supplementary Table S5). The comparison of the qRT-PCR results with the RNA-seq data showed a high correlation coefficient value $\left(R^{2}\right)>0.9$ for the fold change values of the in vivo expression versus the in vitro expression at $3 \mathrm{~h}, 8 \mathrm{~h}$ and $16 \mathrm{~h}$ of growth, respectively (Supplementary Fig. S4). These results demonstrate that the RNA-seq analysis provided overall reliable data.

\section{General characteristics of the transcriptomics of in vivo symbiont cells}

B. insecticola strain RPE64 has a multipartite genome composed of three chromosomes and two plasmids [45]. The overall expression level of genes located on these five genome components is not uniform (Fig. 3a; Supplementary Fig. S5). A principle component analysis (PCA) of the overall transcriptomes clustered the replicates of each condition demonstrating the high reproducibility of the transcriptomes (Fig. 3b). The PCA further revealed a strong partitioning of the conditions, where the transcriptome of the in vivo bacteria was unique compared to the three in vitro transcriptomes (Fig. 3b). The pairwise Pearson correlation coefficients revealed that the in vivo geneexpression profile is relatively similar to that of $3 \mathrm{~h}$-cultured in vitro cells (Fig. 3c). The high correlation of the in vivo cells with lag- and log-phase cells and low correlation with stationary-phase cells is in large part driven by the expression of genes encoding core metabolisms such as cell division, DNA reprication, protein synthesis, and respiration (Fig. 3d).

\section{Cell division, DNA replication, protein synthesis, and respiration}

The genes involved in cell division and DNA replication were highly expressed in the in vivo cells as compared to in vitro stationary cells (Fig. 3d). Furthermore, the genes involved in protein synthesis (i.e. ribosomal subunits, translation factors, and protein chaperones such as GroEL/ GroES and CspA mRNA chaperones) and respiration (i.e.
NADH dehydrogenase, cytochrome $\mathrm{c}$ oxidase and ATP synthetase) were highly expressed in vivo to a similar level as exponentially growing in vitro cells (Fig. 3d). The overall data indicates the active proliferation of in vivo symbionts.

The expression pattern of all annotated genes encoding subunits of the respiratory chain complex I to IV and the ATP synthase and known markers of hypoxia (Supplementary Table S6) is most similar to $3 \mathrm{~h}$ cultures, suggesting that the respiratory activity of in vivo symbionts is high and that these bacteria are not limited by oxygen availability. The $c y o A B C D$ cluster is, however, very lowly expressed in the in vivo bacteria contrary to the culture conditions. Although it is commonly thought that Cyo is used at higher oxygen concentrations, there are also reports that indicate the upregulation and importance of Cyo in low oxygen concentrations [49], including in Burkholderia pseudomallei [50]. In addition, cytochrome bd is also very lowly expressed in in vivo bacteria and this terminal oxidase is known for its high affinity for $\mathrm{O}_{2}$ and its activity under low oxygen concentrations [51]. Furthermore, a previous transcriptome study in B. pseudomallei [50] has identified several genes upregulated under hypoxia conditions, including genes for PHA synthesis, chemotaxis, motility, and some stress response proteins (dps, hsp, usp, and $\operatorname{clp} A B$ ), none of which are induced in in vivo symbionts or many of which are even repressed compared to the culture condition (Supplementary Table S6).

\section{Surface structure: LPS and cell-wall biosyntheses}

In the LPS biosynthesis pathway, genes involved in lipid A, core oligosaccharide (core-OS), 4-amino-4-deoxy-L-arabinose (Ara4N) and O-antigen biosyntheses, and LPS transport were consistently expressed in in vivo cells (Supplementary Fig. S6a, Table S6). In the peptidoglycan biosynthesis pathway, most of the genes, including murA$G, m r a Y, u p p P, m v i N, p b p B$ and $m r d A$, were consistently expressed in vivo (Supplementary Fig. S6b, Table S6). The expression profiles of the LPS and peptidoglycan biosynthesis pathways strongly suggest that intact structures of LPS and cell wall are synthesized in the in vivo condition.

\section{Identification of upregulated and downregulated genes in the in vivo symbiont}

Next, we focused on specific differentially expressed genes between in vivo and in vitro symbiont cells. We compared the in vivo transcriptome with the $3 \mathrm{~h}-, 8 \mathrm{~h}-$, and $16 \mathrm{~h}$-cultured in vitro transcriptomes, and identified 527 in vivospecific upregulated genes and 638 downregulated genes (Supplementary Fig. S7a-b, Tables S7, S8). The 

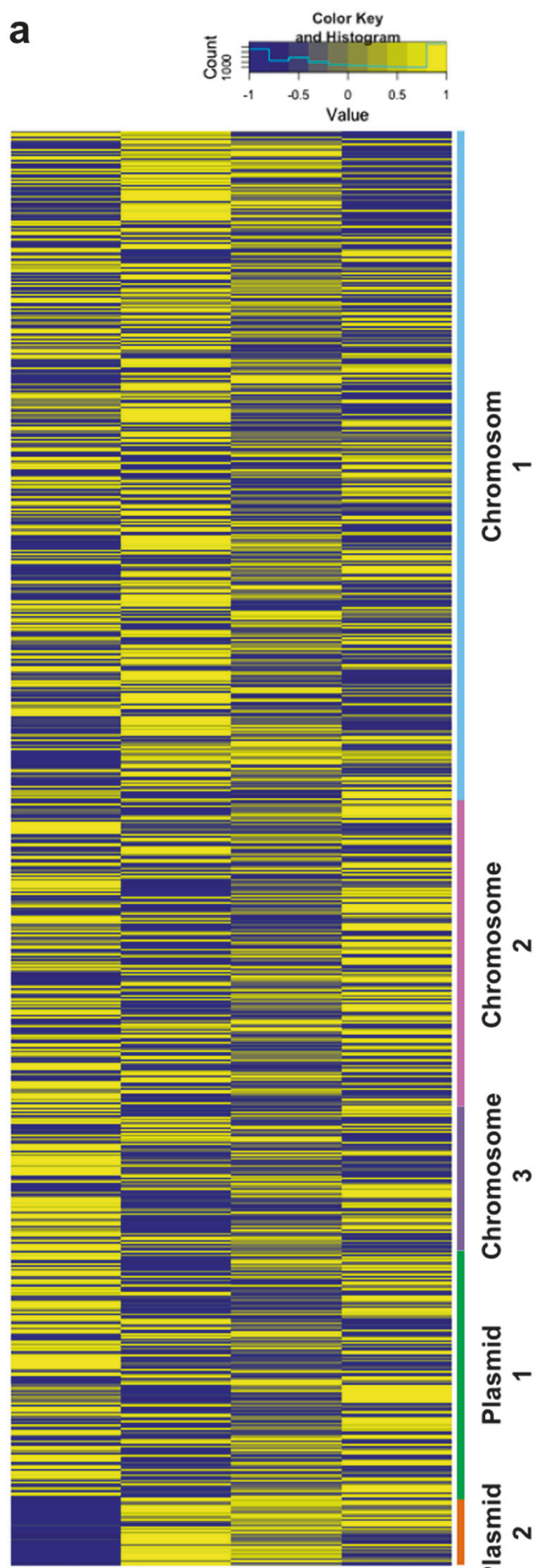

In vivo In vitro In vitro In vitro

$3 \mathrm{~h} \quad 8 \mathrm{~h} \quad 16 \mathrm{~h}$

Fig. 3 Gene expression profiles of in vivo and in vitro Burkholderia symbiont. a heatmap of 5872 gene expression profiles, b Principal component analysis, and $\mathbf{c}$ the pairwise Pearson correlation coefficients of in vivo and $3 \mathrm{~h}-, 8 \mathrm{~h}$-, and $16 \mathrm{~h}$-cultured in vitro cells. In the heatmap, the normalized mean expression level of the three biological

chromosome 2, chromosome 3 , and plasmid 1 contained more in vivo upregulated genes, whereas chromosome 1 and plasmid 2 had more in vivo downregulated genes
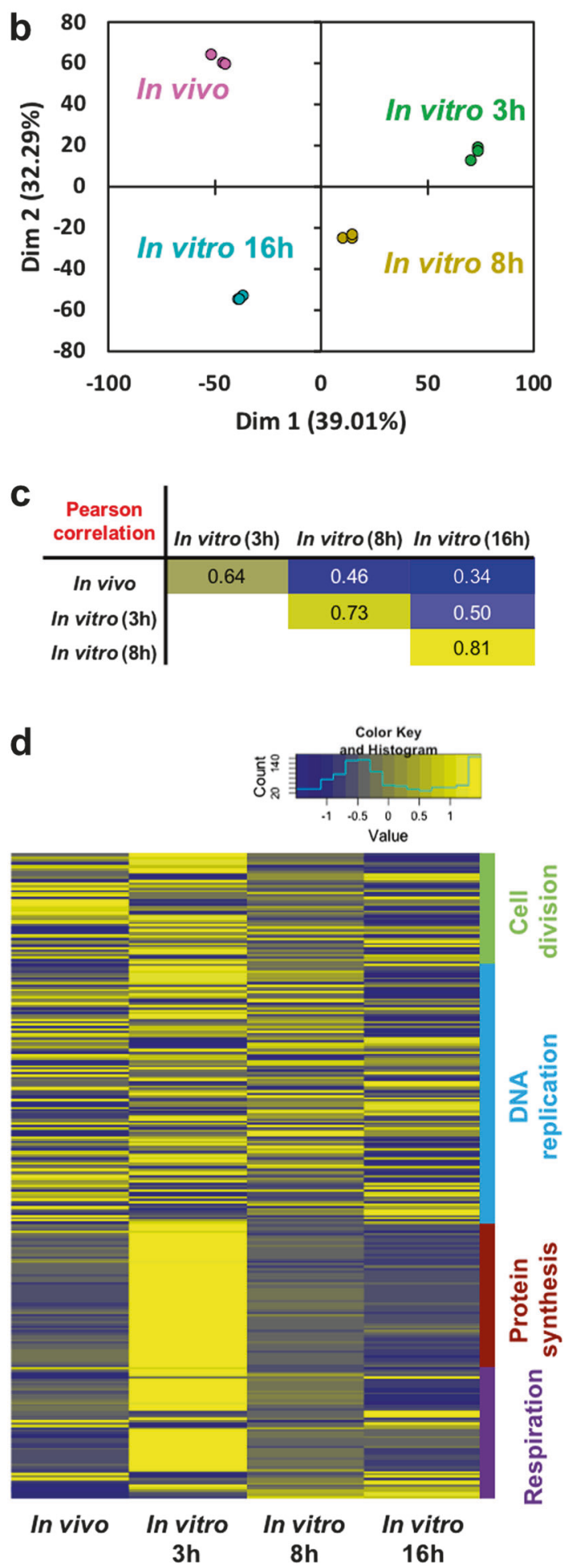

replicates was used, and the color scale from blue to yellow indicates the relative expression level. $\mathbf{d}$ Gene expression profile of core cellular functions. The heatmap shows the expressions of 281 genes encoding cell division, DNA replication, protein synthesis, and respiration in in vivo and in vitro symbiont cells

(Supplementary Fig. S7c). These in vivo upregulated and downregulated genes were classified into clusters of orthologous groups (COG) of the NCBI public database 


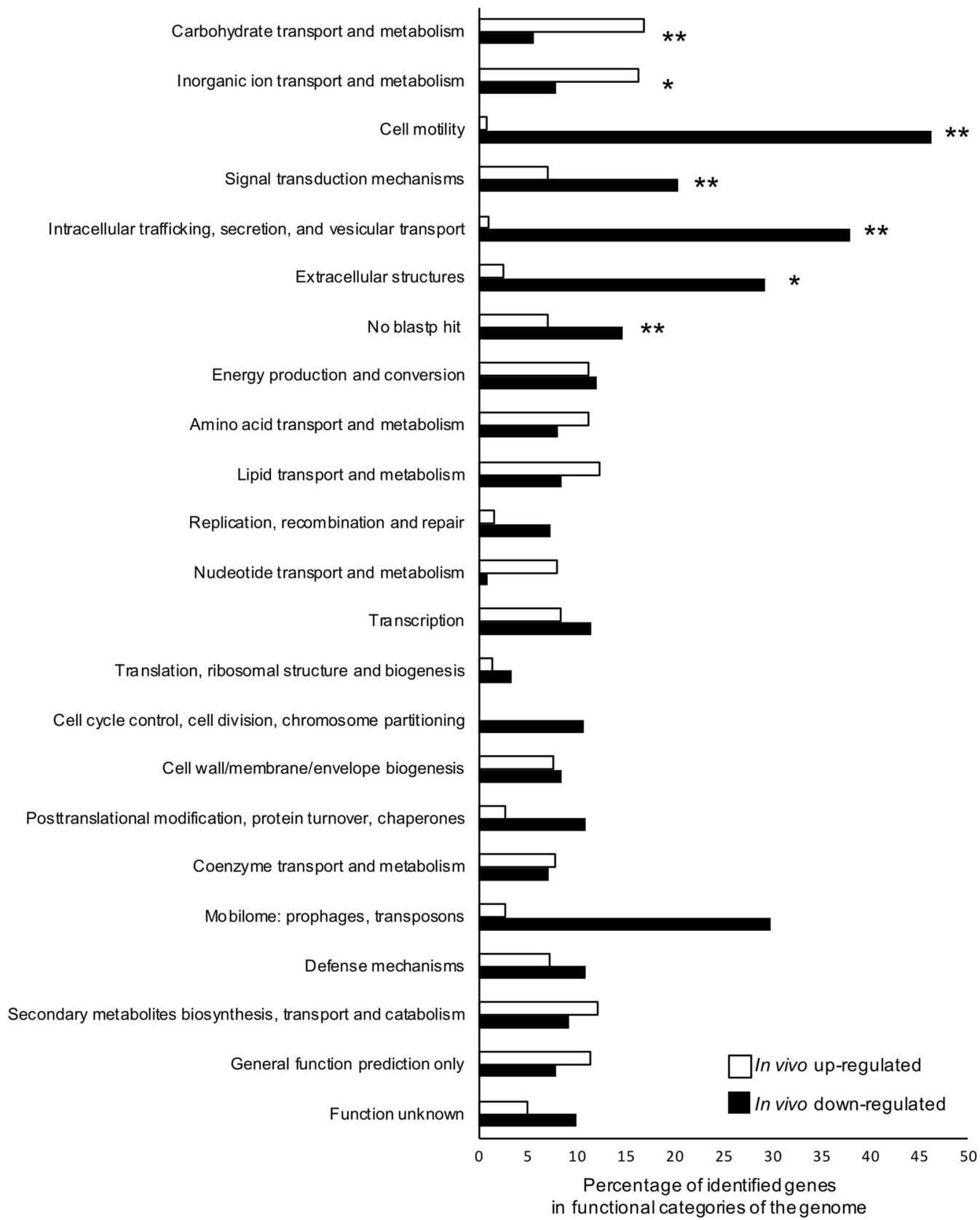

Fig. 4 COG functional classification of in vivo upregulated and downregulated genes. The in vivo upregulated and down-regulated genes were classified using the NCBI COG 2014 database. Asterisks indicate

(Fig. 4). The in vivo upregulated genes were enriched mainly in two COG categories: "carbohydrate transport and metabolism" and "inorganic ion transport and metabolism". In contrast, the in vivo downregulated genes were significantly enriched in five COGs: "cell motility", "signal transduction mechanism", "intracellular trafficking", "secretion and vesicular transport", "extracellular structures", and "no blastp hit". In all COGs except cell motility, over $60 \%$ genes were equally expressed in in vivo and in vitro cells (Supplementary Fig. S8). statistically significant difference between in vivo and in vitro cells (Fisher's exact test with Bonferroni correction; $* P<0.05, * * P<0.01$ )

A detailed landscape of in vivo metabolic alterations was obtained by classifying in vivo upregulated and downregulated genes with the KEGG (Kyoto Encyclopedia of Genes and Genomes) database. In the KEGG database, 204 in vivo upregulated genes and 196 down-regulated genes were annotated (Fig. 5; Supplementary Tables S7, S8). The in vivo upregulated and down-regulated genes/pathways are described in more detail in the following sections. 


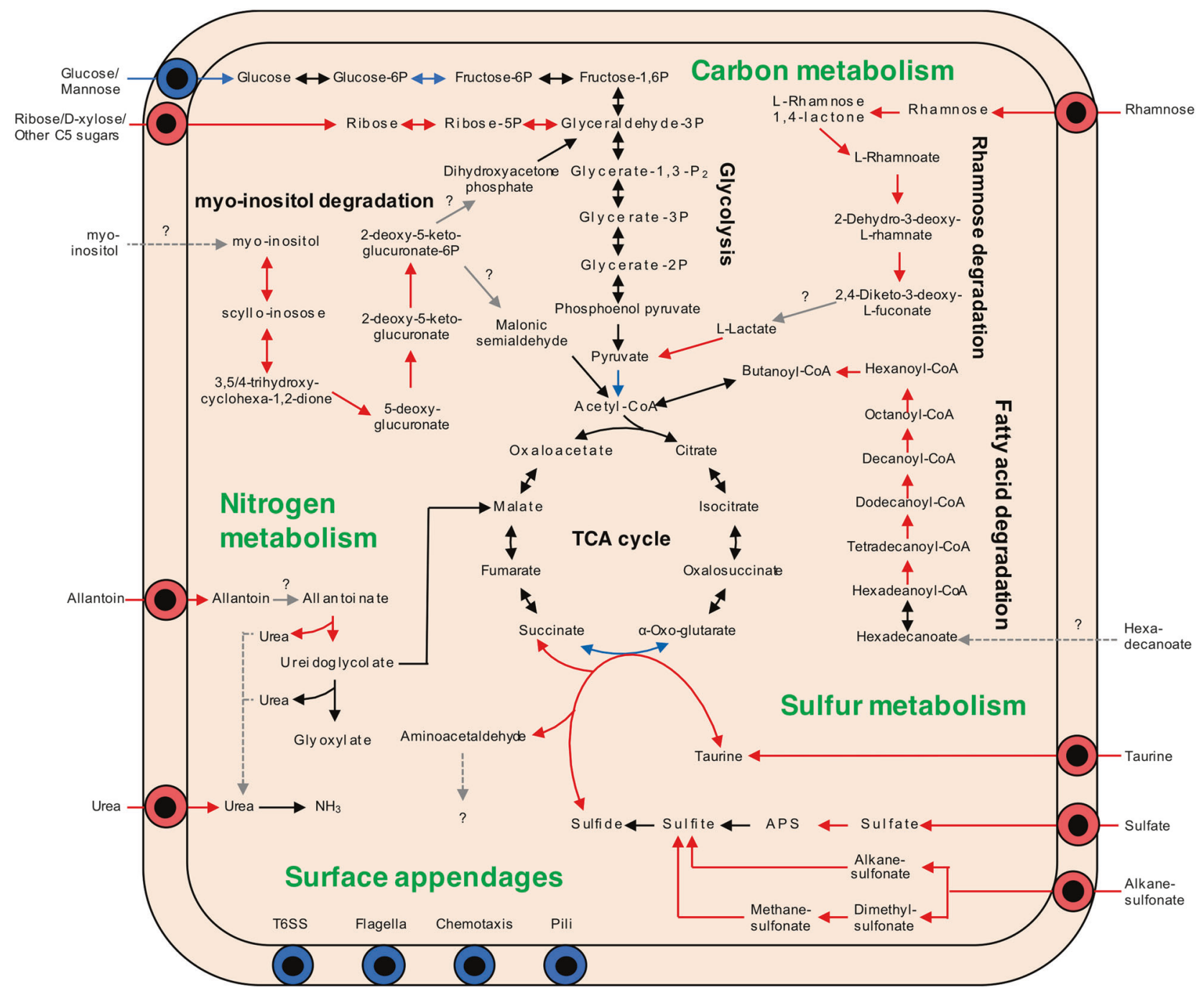

Fig. 5 Overview of KEGG metabolic pathways in in vivo upregulated and down-regulated genes. Arrows indicate metabolic direction, and the color shows gene expression levels in RNA-seq: red, in vivo upregulatd; blue, in vivo down-regulated; black, un-changed expression levels

\section{Upregulated pathways in in vivo Burkholderia symbiont}

\section{Carbon metabolism}

The transcriptome comparisons clearly demonstrated that in vivo cells strongly uptake and assimilate pentose sugars like ribose and rhammose, as well as myo-inositol as carbon sources (Fig. 5; Supplementary Table S6). On the other hand, the glucose transporters and the pathway upstream of glycolysis, which was highly expressed in vitro, were downregulated. Furthermore, in contrast to the downregulation of the fatty acid biosynthesis pathway (Supplementary Table S6), the fatty acid degradation pathway was consistently and strongly upregulated in vivo (Fig. 5; Supplementary Table S6). These results strongly suggest that these sugars and fatty acid are carbon sources provided by the host insect.

\section{Nitrogen metabolism}

In the in vivo symbiont, transporters and assimilation pathways of allantoin and urea were specifically upregulated (Fig. 5; Supplementary Fig. S9a, Table S6). Most insects excrete uric acid as nitrogen waste and some species, including heteropteran bugs, convert it to allantoin or further to allantoic acid [52, 53]. In addition, although the ornitine cycle has not been determined in insects, urea is consistently detected in the hemolymph in diverse insects $[54,55]$. The strong gene induction of the allantoin and urea pathways indicates that the Burkholderia symbiont utilizes these host nitrogen wastes inside the midgut crypts. 


\section{Sulfur metabolism}

The in vivo symbiont highly and specifically expressed the sulfate reduction pathway and degradation pathway of sulfonates such as taurine and alkanesulphonate (Fig. 5; Supplementary Fig. S9b, Table S6). Inside the symbiont, sulfate could be assimilated to cysteine. Among the sulfur metabolism pathways, a taurine dioxigenase, $\operatorname{tau} D$, was strongly upregulated (Supplementary Fig. S9b), which converts taurine and $\alpha$-oxoglutarate to sulfite, succinate, and aminoacetaldehyde [56].

Other upregulated metabolisms are described in Supplementary Information.

\section{Downregulated pathways in in vivo Burkholderia symbiont}

The in vivo downregulated genes or pathways are involved in cell motility, chemotaxis, and type VI secretion system (see Supplementary Information). Remarkably, gene expression of the plasmid 2 was almost completely suppressed in vivo (Fig. 3a; Supplementary Fig. S5). We found that this was because of a frequent loss of this plasmid in the in vivo symbiont (see Supplementary Information).

\section{Biosynthesis pathways of essential amino acids and B vitamins}

We specifically analyzed biosynthesis genes of the essential amino acids and B vitamins, because these are potentially key metabolites in the stinkbug-Burkholderia symbiosis as it is the case in other nutrional symbioses of phytophagous insects. The biosynthesis pathways for the nine essential amino acids were not among the identified upregulated genes in the in vivo symbiont cells, but the genes were nevertheless highly expressed both in vivo and in vitro (Supplementary Fig. S10, Table S6). Similarly, biosynthesis pathways for B vitamins (i.e. thiamin, riboflavin, niacin, pantothenic acid, pyridoxine, cobalamin, folic acid, and biotin), including a few genes not annotated in the KEGG database, were highly expressed both in vivo and in vitro (Supplementary Fig. S11, Table S6).

\section{Discussion}

In general, the bacterial cell morphology strongly depends on environmental cues, such as the nutritional condition and AMP stress [57-59]. The smaller cell size and reduced DNA content of the in vivo symbiont (Fig. 1) could be interpreted as an indication that these bacteria are starved and that the midgut internal environment is nutritionallypoor or -biased for the symbiotic Burkholderia
(Supplementary Fig. S1). However, the transcriptome analysis tells us a different message: the overall gene expression profile of the midgut bacteria is similar to the profile of exponentially growing in vitro cells rather than stationary phase ones, wherein genes involved in core cellular functions, such as cell division, protein biosyntheses, and respiration are highly expressed in the in vivo symbiont cells (Fig. 3d). The expression profiles further suggest that the symbiotic bacteria are not in a microaerobic environment. Thus, it is unlikely that nutrional or oxygen limitation provokes the specific morphology and cytology of the Burkholderia and therefore, other factors of the gut environment could determine the phenotype of the in vivo symbiont cells.

Morphological alterations have been described in other host-bacterium interactions. In the case of intracellular infection of macrophages by Salmonella, AMPs produced by the macrophages inhibit the division of the Salmonella cells and their continuous growth leads to filamentation of the bacteria [60]. Another well-known example is found in the legume-Rhizobium symbiosis, in which the Rhizobium cells become enlarged and polyploid because of the suppression of cell division by AMPs, called Nodule-specific Cysteine-Rich peptides (NCRs), inducing their transition into a unique cell-state called the bacteroid, which is specialized to fix atmospheric dinitrogen [61-63]. Interestingly, also the midgut crypts of $R$. pedestris produce AMP-like cationic peptides, called CCRs. More than 90 different genes encoding such CCR peptides are highly expressed by the midgut crypts and most of them are only induced when the crypts are colonized [39]. Although the biological function of the CCRs remains unclear, in this study we demonstrated that the chemically synthesized CCR0008 and CCR0480 have an antibiotic and growth inhibitory activity against the symbiont (Fig. 2; Supplementary Table S3), suggesting that CCRs may be involved in controlling the Burkholderia population in the gut. The production of this CCR peptide family by the midgut crypts is analogous to the NCR peptides produced in the legume-Rhizobium symbiosis and it will be of interest in the future to determine whether these peptides affect the bacterial cell cycle, DNA replication, or cell morphology.

Another notable feature of the in vivo symbiont is the high susceptibility to diverse stresses (Fig. 2). Previous studies have reported that cell surface structures are altered in in vivo Burkholderia symbionts and in particular that the O-antigen moiety of LPS is lost while the coreoligosaccharide structure is required to maintain a stable colonization [36, 37]. In addition, their cell envelope shows perturbations in the form of outer membrane blebs (Fig. 1k; Supplementary Fig. S2) [36]. These blebs could be emerging outer membrane vesicles (OMVs), which are commonly produced by Gram-negative bacteria enabling them 
to deal with certain stress conditions [64]. Similar type of blebs and OMVs have been reported frequently to be produced by bacteria in response to antimicrobial peptides [6569], including to the NCR peptides [62]. Thus, these blebs could be the result of the exposure of the in vivo symbiont to the CCR peptides. The susceptibility of the in vivo symbiont to diverse stresses could be largely explained by these surface alterations.

Although only the core-oligosaccharide is maintained in the in vivo symbiont and the O-antigen is absent [37], we found that both $\mathrm{O}$-antigen and core-oligosaccharide biosynthesis pathway genes were expressed in the midgut bacteria (Supplementary Table S6). This contradiction between the transcriptome and biochemical data suggest that the synthesis of these surface structures is posttranscripionally affected by host factor(s). These could be glycolytic enzymes, inhibitors of O-antigen translation and transport, and/or AMPs attacking bacterial surface (such as CCRs) in the midgut crypts.

We further find that the gene cluster involved in the Ara4N modification of lipid A is constitutively expressed in all the tested conditions. This modification is known in some bacteria to be introduced onto lipid A upon sensing of AMPs [70-72], which renders lipid A less charged and therefore less vulnerable to AMPs. However, in Burkholderia, this modification is probably constitutively added to lipid A, which is essential for viability in B. cenocepacia [73]. If the same is true for $B$. insecticola, it is not surprising the constitutive expression of genes for the Ara4N modification of lipid A and therefore, it is unlikely that the increased sensitivity of the in vivo bacteria is due to the loss of the Ara4N modification.

The RNA-seq comparisons provided indications on the nutrients that are probided by the bean bug to the Burkholderia symbiont. In the midgut crypts, the host likely provides ribose, rhamnose, myo-inositol, and fatty acids as carbon sources, allantoin and urea as nitrogen sources, and sulfate, and sulfonates as sulfur sources (Fig. 5). Allantoin is the major nitrogen waste compound in heteropteran insects $[52,53]$, and urea is probably a nitrogen waste as well $[54,55]$. Considering the highly upregulation of the allantoin and urea pathways (Fig. 5; Supplementary Fig. S9a), the recycling of nitrogen waste may be a biological function of the Burkholderia symbiont in the bean bug. Recycling of host metabolic waste is one of the common traits in insect symbionts $[6,74]$. Such a "recycling" symbiosis could enable the host to more efficiently utilize nutrients from food and to save energy, resulting in the more rapid growth and larger body size of the symbiotic bean bugs.

The other intriguing observation is the sulfer metabolism of the Burkholderia symbiont. In general, insects do not have a sulfate-reducing enzyme and sulfate has to be reduced to sulfite that can be assimilated [75], while our RNA-seq results revealed that the in vivo symbiont can assimilate sulfate to cysteine. It is notable that genes involved in sulfate assimilation have been reported in other symbiotic bacteria in insects [76-79], suggesting the sulfate assimilation is a common feature of insect symbionts. Furthermore, another notable point here is that the taurine degradation is tightly linked to the TCA cycle, wherein tauD provides succinate by oxidising taurine and $\alpha$ oxoglutarate [56]. Since the genes involved in the conversion from $\alpha$-oxoglutarate to succinate in the TCA cycle are strongly suppressed in vivo (Fig. 5; Supplementaly Table S6), the taurine metabolism appears likely to complement this conversion process, which may enable the host to control the symbiont via taurine feeding.

In many insects, the nutritional benefit of symbionts is the provisioning of essential amino acids and/or B vitamins $[80,81]$. A recent physiological study on the gut bacteria of a cotton pest, Dysdercus fasciatus (Heteroptera: Pyrrhocoridae), has revealed that the symbiotic bacteria provide B vitamins [82], which are deficient in cotton seeds. Unlike cotton seeds, soybean seeds, the food of $R$. pedestris, are known to be rich in $\mathrm{B}$ vitamins except vitamin $\mathrm{B}_{12}$ (cobalamin) (Supplementary Fig. S12) [83, 84]. Biosynthetic pathways for all 8 kinds of $\mathrm{B}$ vitamins were highly expressed in the in vivo condition, suggesting the possibility that the symbiont provide the host insect with B vitamins, particularly vitamin $B_{12}$. Although soybean seeds are rich in amino acids, two essential amino acids, methionine, and tryptophan, are limited (Supplementary Fig. S12) [83, 84]. The Burkholderia symbiont has retained intact pathways for producing these essential amino acids and the involved genes were highly expressed in vivo, suggesting that the Burkholderia symbiont mainly supplies methionine and tryptophan to the host.

How exactly does the host extract the nutrients derived from the Burkholderia symbiont? It seems that an excess of symbionts in the midgut crypts is not excreted since $R$. pedestris excrements do not contain the symbiont [25], but that they instead flow into the M4B region [40], wherein AMPs and digestive enzymes like cathepsins are highly and specifically expressed [39] and kill the bacteria [40, 41]. Furthermore, in dissected midguts infected with GFPexpressing Burkholderia and stained with PI, we noticed that the bacteria in the M4B region close to the M4 are still alive, GFP-positive and PI-negative, while in the more distant regions of M4B the GFP-signal disappears suddenly and PI staining becomes strong (Fig. 6a). This suggests that whole symbiont cells are digested in the M4B region and that the liberated nutrients are absorbed by the host insect. Based on these biological features of the in vivo symbiont cells, we here propose the following in vivo life cycle of the Burkholderia symbiont (Fig. 6b): (1) after colonization in 
a

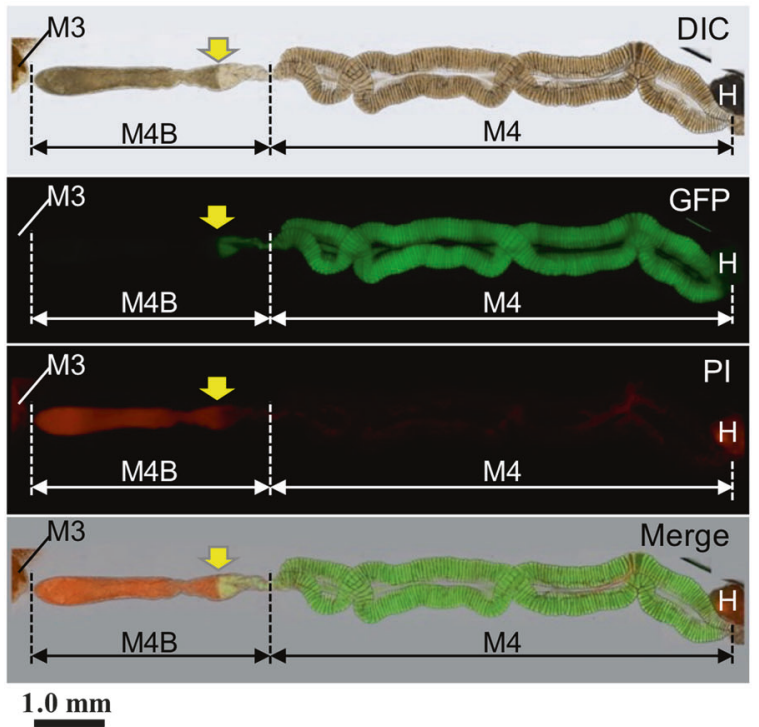

b

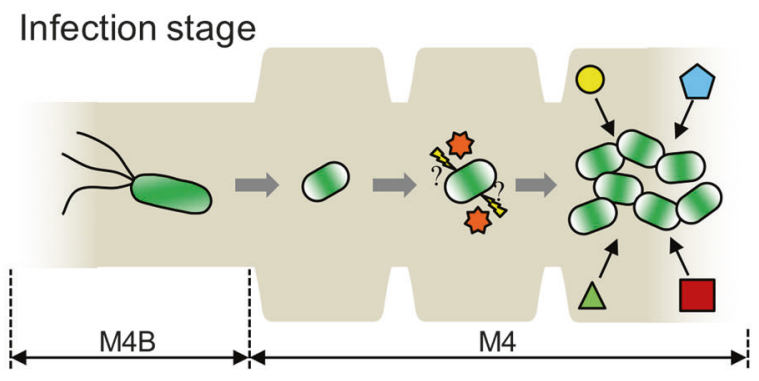

Mature colonization stage

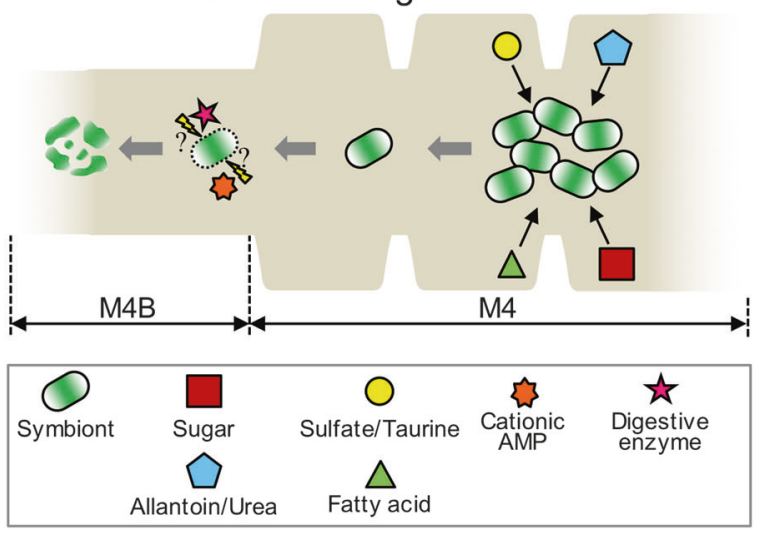

the midgut crypts, the symbiont actively proliferates by use of host-provided nutrients including metabolic wastes such as allantoin; (2) excess symbiont cells flow into M4B and are digested by the host, wherein the backward flow of the symbiotic bacteria from M4 to M4B could be made possible by a temporal and local regain of motility of the symbiont, by pressure generated by the multiplication of the symbiont in M4, or by gut peristaltic movements; (3) not a specific nutrient but the ensemble of nutrients of the symbiont cells
4Fig. 6 A hypothetical model for the life cycle of the Burkholderia symbiont in the midgut of $R$. pedestris. a The PI staining in the midgut of 5th instar nymph infected with the strain RPE225 (a GFPexpressing mutant). DIC, GFP fluorescence, PI fluorescence, and merged images are shown. Arrows indicate the border of GFP and PI fluorescent signals. M3, midgut 3rd section; M4, midgut 4th section (crypts); M4B, M4 bulb; H, hindgut. A red auto-fluorescence was observed in M3 and H. b Graphical summary of Burkholderia features and midgut functions of $R$. pedestris. (Upper) During the infection stage of Burkholderia symbiont at the insect midgut, the Burkholderia symbiont cells lose flagellar motility and modify their envelope under the influence of host stress factors such as cationic AMPs (e.g. CCRs), they proliferate by metabolizing host waste materials, such as sulfate and allantoin. (Lower) In the mature colonization stage, these symbiont cells are digested in the M4B section mediated by host factors such as cathepsin proteases and CCRs, and the host absorbs nutrients derived from whole-bacterial cells

is absorbed, contributing to the rapid growth and enhanced reproduction of the bean bug host. Such a symbiotic association, i.e. a host cultivates and consumes whole cells of its symbiont, has been reported in other animal-microbe symbiotic systems, such as the cultivation symbiosis in leafcutting ants [85] and the gut symbiosis in herbivorous rodents that show a coprophagy behavior [86, 87].

\section{Conclusion and perspective}

In this study, we demonstrated how the Burkholderia symbiont copes with the environment of the midgut crypts, and suggested what roles the symbiont plays in the symbiotic organ. To understand the host-symbiont nutritional interactions in the stinkbug-Burkholderia association more deeply, metabolomics of the midgut crypts and feces, and/or isotope-labeling and metabolite-tracing experiments will be needed. These experiments would also elucidate the mechanisms that enable the bean bug to obtain a bigger body size and produce a higher egg number in the presence of the Burkholderia symbionts. Furthermore, it should be noted that almost $25 \%$ of the in vivo upregulated genes are functionally unknown, which underscores how much the endosymbiosis between the bean bug and Burkholderia is still enigmatic. Nevertheless, this report about the bacterial features in the bean bug is important to orient future work on this model symbiosis.

Acknowledgements Part of this work was performed at the Open Facility of Hokkaido University and the Imagerie-Gif facilities. This work has benefited from the expertise of the High-throughput Sequencing Platform of I2BC and from support by the Labex 'Saclay Plant Sciences' (ANR-11-IDEX-0003-02). This work was supported by the MEXT KAKENHI (15H05638 to Y.K.), the JSPS Research Fellowship for Young Scientists (14J03996 and 20170267 to T.O.) and the JSPS Invitation Fellowship for Research in Japan (L14556 to P.M.). 
Author contributions TO, PM, and YK designed the project. TO, TS, and PM performed the stress tests. MT and QB conducted flow cytometry analyses. XYM and YM performed electron microscopy. TO, RF, SS, TF, FL, KT, and PM conducted RNA-seq and analyzed transcriptome data. TO, PM, and YK wrote the manuscript. All coauthors edited the manuscript before submission.

\section{Compliance with ethical standards}

Conflict of interest The authors declare that they have no conflict of interest.

Publisher's note: Springer Nature remains neutral with regard to jurisdictional claims in published maps and institutional affiliations.

\section{References}

1. Schwartzman JA, Ruby EG. Stress as a normal cue in the symbiotic environment. Trends Microbiol. 2016;24:414-24.

2. Yang DC, Blair KM, Salama NR. Staying in shape: the impact of cell shape on bacterial survival in diverse environments. Microbiol Mol Biol Rev. 2016;80:187-203.

3. Buchner P. Endosymbiosis of animals with plant microorganims. New York: Interscience; 1965.

4. Bourtzis K, Miller T. Insect symbiosis. Boca Raton, FL: CRC Press; 2003.

5. Kikuchi Y. Endosymbiotic bacteria in insects: their diversity and culturability. Microbes Environ. 2009;24:195-204.

6. Ankrah NYD, Douglas AE. Nutrient factories: metabolic function of beneficial microorganisms associated with insects. Environ Microbiol. 2018;20:2002-11.

7. McCutcheon JP, Moran NA. Extreme genome reduction in symbiotic bacteria. Nat Rev Microbiol. 2011;10:13-26.

8. Utami YD, Kuwahara H, Murakami T, Morikawa T, Sugaya K, Kihara K, et al. Phylogenetic diversity and single-cell genome analysis of "Melainabacteria", a non-photosynthetic cyanobacterial group, in the termite gut. Microbes Environ. 2018;33:50-57.

9. Noda S, Shimizu D, Yuki M, Kitade O, Ohkuma M. Hostsymbiont cospeciation of termite-gut cellulolytic protists of the genera Teranympha and Eucomonympha and their Treponema endosymbionts. Microbes Environ. 2018;33:26-33.

10. Salem H, Bauer E, Kirsch, Berasategui A, Cripps M, Weiss B, et al. Drastic genome reduction in an herbivore's pectinolytic symbiont. Cell. 2017;171:1520-31.

11. Sabree ZL, Kambhampati S, Moran NA. Nitrogen recycling and nutritional provisioning by Blattabacterium, the cockroach endosymbiont. Proc Natl Acad Sci USA. 2009;106:19521-6.

12. Kaltenpoth M. Actinobacteria as mutualists: general healthcare for insects? Trends Microbiol. 2009;17:529-35.

13. Flórez LV, Biedermann PH, Engl T, Kaltenpoth M. Defensive symbioses of animals with prokaryotic and eukaryotic microorganisms. Nat Prod Rep. 2015;32:904-36.

14. Werren JH, Baldo L, Clark ME. Wolbachia: master manipulators of invertebrate biology. Nat Rev Microbiol. 2008;6:741.

15. Itoh H, Tago K, Hayatsu M, Kikuchi Y. Detoxifying symbiosis: microbe-mediated detoxification of phytotoxins and pesticides in insects. Nat Prod Rep. 2018;35:434-54.

16. Bright $\mathrm{M}$, Bulgheresi S. A complex journey: transmission of microbial symbionts. Nat Rev Microbiol. 2010;8:218-30.

17. Baumann P. Biology of bacteriocyte-associated endosymbionts of plant sap-sucking insects. Annu Rev Microbiol. 2005;59:155-89.

18. Mergaert P, Kikuchi Y, Shigenobu S, Nowack ECM. Metabolic integration of bacterial endosymbionts through antimicrobial peptides. Trends Microbiol. 2017;25:703-12.
19. Kadosawa T, Santa H. Growth and reproduction of soybean pod bugs (Heteroptera) on seeds of legumes. Bull Chugoku Nat Agric Exp Stn. 1981;19:75-97.

20. Kono S. Ecological studies of stink bugs injuring soybean seeds for developing effective control measures. Sp Rep Hyogo Agric Exp Stn. 1991;16:32-68.

21. Schaefer CW, Panizzi AR. Heteroptera of economic importance. Boca Raton, FL: CRC press; 2000.

22. Kikuhara Y. The Japanese species of the genus Riptortus (Heteroptera, Alydidae) with description of a new species. Jpn J Syst Entomol. 2005;11:299-311.

23. Takeshita K, Tamaki H, Ohbayashi T, Meng XY, Sone T, Mitani $\mathrm{Y}$, et al. Burkholderia insecticola sp. nov., a gut symbiotic bacterium of the bean bug Riptortus pedestris. Int J Syst Evol Microbiol. 2018;68:2370-4.

24. Takeshita K, Kikuchi Y. Riptortus pedestris and Burkholderia symbiont: an ideal model system for insect-microbe symbiotic associations. Res Microbiol. 2016;168:175-87.

25. Kikuchi Y, Hosokawa T, Fukatsu T. Insect-microbe mutualism without vertical transmission: a stinkbug acquires a beneficial gut symbiont from the environment every generation. Appl Environ Microbiol. 2007;73:4308-16.

26. Kikuchi Y, Fukatsu T. Live imaging of symbiosis: spatiotemporal infection dynamics of a GFP-labelled Burkholderia symbiont in the bean bug Riptortus pedestris. Mol Ecol. 2014;23:1445-56.

27. Lee JB, Park KE, Lee SA, Jang SH, Eo HJ, Jang HA, et al. Gut symbiotic bacteria stimulate insect growth and egg production by modulating hexamerin and vitellogenin gene expression. Dev Comp Immunol. 2017;69:12-22.

28. Kikuchi Y, Hosokawa T, Fukatsu T. Specific developmental window for establishment of an insect-microbe gut symbiosis. Appl Environ Microbiol. 2011;77:4075-81.

29. Kuechler SM, Matsuura Y, Dettner K, Kikuchi Y. Phylogenetically diverse Burkholderia associated with midgut crypts of spurge bugs, Dicranocephalus spp. (Heteroptera: Stenocephalidae). Microbes Environ. 2016;31:145-53.

30. Kikuchi Y, Yumoto I. Efficient colonization of the bean bug Riptortus pedestris by an environmentally transmitted Burkholderia symbiont. Appl Environ Microbiol. 2013;79:2088-91.

31. Kim JK, Lee BL. Symbiotic factors in Burkholderia essential for establishing an association with the bean bug, Riptortus pedestris. Arch Insect Biochem Physiol. 2015;88:4-17.

32. Ohbayashi T, Takeshita K, Kitagawa W, Nikoh N, Koga R, Meng $\mathrm{XY}$, et al. Insect's intestinal organ for symbiont sorting. Proc Natl Acad Sci USA. 2015;112:E5179-E5188.

33. Kinosita Y, Kikuchi Y, Mikami N, Nakane D, Nishizaka T. Unforeseen swimming and gliding mode of an insect gut symbiont, Burkholderia sp. RPE64, with wrapping of the flagella around its cell body. ISME J. 2018;12:838-48.

34. Kim JK, Lee HJ, Kikuchi Y, Kitagawa W, Nikoh N, Fukatsu T, et al. Bacterial cell wall synthesis gene uppP is required for Burkholderia colonization of the stinkbug gut. Appl Environ Microbiol. 2013;79:4879-86.

35. Kim JK, Won YJ, Nikoh N, Nakayama H, Han SH, Kikuchi Y, et al. Polyester synthesis genes associated with stress resistance are involved in an insect-bacterium symbiosis. Proc Natl Acad Sci USA. 2013;110:E2381-2389.

36. Kim JK, Son DW, Kim CH, Cho JH, Marchetti R, Silipo A, et al. Insect gut symbiont susceptibility to host antimicrobial peptides caused by alteration of the bacterial cell envelope. J Biol Chem. 2015;290:21042-53.

37. Kim JK, Jang HA, Kim MS, Cho JH, Lee JB, Lorenzo FD, et al. The lipopolysaccharide core oligosaccharide of Burkholderia plays a critical role in maintaining a proper gut symbiosis with the bean bug Riptortus pedestris. J Biol Chem. 2017;292:19226-37. 
38. Jang SH, Jang HA, Lee J, Kim JU, Lee SA, Park KE, et al. PhaR, a negative regulator of $\mathrm{PhaP}$, modulates the colonization of a Burkholderia gut symbiont in the midgut of the host insect, Riptortus pedestris. Appl Environ Microbiol. 2017;83:e0459-17.

39. Futahashi R, Tanaka K, Tanahashi M, Nikoh N, Kikuchi Y, Lee $\mathrm{BL}$, et al. Gene expression in gut symbiotic organ of stinkbug affected by extracellular bacterial symbiont. PLOS ONE. 2013;8: e64557.

40. Kim JK, Kim NH, Jang HA, Kikuchi Y, Kim CH, Fukatsu T, et al. Specific midgut region controlling the symbiont population in an insect-microbe gut symbiotic association. Appl Environ Microbiol. 2013;79:7229-33.

41. Byeon JH, Seo ES, Lee JB, Lee MJ, Kim JK, Yoo JW, et al. A specific cathepsin-L-like protease purified from an insect midgut shows antibacterial activity against gut symbiotic bacteria. Dev Comp Immunol. 2015;53:79-84.

42. Jang HA, Seo ES, Seong MY, Lee BL. A midgut lysate of the Riptortus pedestris has antibacterial activity against LPS Oantigen-deficient Burkholderia mutants. Dev Comp Immunol. 2016;67:97-106.

43. Martin M. Cutadapt removes adapter sequences from highthroughput sequencing reads. EMBnet J. 2011;17:10-12.

44. Li H, Durbin R. Fast and accurate short read alignment with Burrows-Wheeler transform. Bioinformatics. 2009;25:1754-60.

45. Shibata TF, Maeda T, Nikoh N, Yamaguchi K, Oshima K, Hattori $\mathrm{M}$, et al. Complete genome sequence of Burkholderia sp. strain RPE64, bacterial symbiont of the bean bug Riptortus pedestris. Genome Announc. 2013;1:e00441-13.

46. Love MI, Huber W, Anders S. Moderated estimation of fold change and dispersion for RNA-seq data with DESeq2. Genome Biol. 2014;15:550.

47. Lê S, Josse J, Husson F. FactoMineR: an R package for multivariate analysis. J Stat Softw. 2008;25:1-18.

48. Lee JB, Byeon JH, Jang HA, Kim JK, Yoo JW, Kikuchi Y, et al. Bacterial cell motility of Burkholderia gut symbiont is required to colonize the insect gut. FEBS Lett. 2015;589:2784-90.

49. Lunak ZR, Noel KD. A quionol oxidase, encoded by $c y o A B C D$, is utilized to adapt to lower $\mathrm{O}_{2}$ concentrations in Rhizobium etli CFN42. Microbiol . 2015;161:203-12.

50. Hamad MA, Austin CR, Stewart AL, Higgins M, Vazquez-Torres A, Voskuil MI. Adaptation and antibiotic tolerance of anaerobic Burkholderia pseudomallei. Antimicrob Agents Chemother. 2011;55:3313-23.

51. Borisov VB, Gennis RB, Hemp J, Verkhovsky MI. The cytochrome $b d$ respiratory oxygen reductases. Biochim Biophys Acta. 2011;1807:1398-413.

52. Bursell E. The excretion of nitrogen in insects. Adv Insect Physiol. 1967;4:33-67.

53. Cochran DG. Nitrogen excretion in cockroaches. Annu Rev Entomol. 1985;30:29-49.

54. Corrigan JJ. Nitrogen metabolism in insects. In: Campbell JW editors. The Comparative Biochemistry of Nitrogen Metabolism: The invertebrates. 1st edn. New York, USA: Academic Press; 1970. p. 388-488.

55. Mullins D. Chemistry and physiology of the hemolymph. In: Kerkut GA, Gilbert LI editors. Comprehensive Insect Physiology, Biochemistry and Pharmacology. 3rd edn. London, UK: Pergamon; 1985. p. 356-400.

56. Eichhorn E, van der Ploeg JR, Kertesz MA, Leisinger T. Characterization of $\alpha$-ketoglutarate-dependent taurine dioxygenase from Escherichia coli. J Biol Chem. 1997;272:23031-6.

57. Young KD. The selective value of bacterial shape. Microbiol Mol Biol Rev. 2006;70:660-703.

58. Young KD. Bacterial morphology: why have different shapes? Curr Opin Microbiol. 2007;10:596-600.
59. Willis L, Huang KC. Sizing up the bacterial cell cycle. Nat Rev Microbiol. 2017;15:606

60. Rosenberger CM, Gallo RL, Finlay BB. Interplay between antibacterial effectors: a macrophage antimicrobial peptide impairs intracellular Salmonella replication. Proc Natl Acad Sci USA. 2004;101:2422-7.

61. Czernic P, Gully D, Cartieaux F, Moulin L, Guefrachi I, Patrel D, et al. Convergent evolution of endosymbiont differentiation in dalbergioid and inverted repeat-lacking clade legumes mediated by nodule-specific cysteine-rich peptides. Plant Physiol. 2015;169:1254-65.

62. Montiel J, Downie JA, Farkas A, Bihari P, Herczeg R, Balint B, et al. Morphotype of bacteroids in different legumes correlates with the number and type of symbiotic NCR peptides. Proc Natl Acad Sci USA. 2017;114:5041-6.

63. Mergaert P. Role of antimicrobial peptides in controlling symbiotic bacterial populations. Nat Prod Rep. 2018;35:336-56.

64. Schwechheimer C, Kuehn MJ. Outer-membrane vesicles from Gram-negative bacteria: biogenesis and functions. Nat Rev Microbiol. 2015;13:605-19.

65. Manning AJ, Kuehn MJ. Contribution of bacterial outer membrane vesicles to innate bacterial defense. BMC Microbiol. 2011;11:258

66. Duperthuy M, Sjöström AE, Sabharwal D, Damghani F, Uhlin BE, Wai SN. Role of the Vibrio cholerae matrix protein Bap1 in cross-resistance to antimicrobial peptides. PLoS Pathog. 2013;9: e1003620.

67. Kulkarni HM, Swamy ChVB, Jagannadham MV. Molecular characterization and functional analysis of outer membrane vesicles from the Antarctic bacterium Pseudomonas syringae suggest a possible response to environmental conditions. J Proteome Res. 2014; $13: 1345-1358$.

68. Chileveru HR, Lim SA, Chairatana P, Wommack AJ, Chiang I-L, Nolan EM. Visualizing attack of Escherichia coli by the antimicrobial peptide Human Defensin 5. Biochemistry. 2015;54:1767-1777.

69. Urashima A, Sanou A, Yen H, Tobe T. Enterohaemorrhagic Escherichia coli produces outer membrane vesicles as an active defence system against antimicrobial peptide LL-37. Cell Microbiol. 2017;19:e12758.

70. Raetz CRH, Reynolds CM, Trent MS, Bishop RE. Lipid A modification systems in Gram-negative bacteria. Annu Rev Biochem. 2007;76:295-329.

71. Chen HD, Groisman EA. The biology of the PmrA/PmrB twocomponent system: the major regulator of lipopolysaccharide modifications. Annu Rev Microbiol. 2013;67:83-112.

72. Dalebroux ZD, Miller SI. Salmonellae PhoPQ regulation of the outer membrane to resist innate immunity. Curr Opin Microbiol. 2014;17:106-13.

73. Ortega XP, Cardona ST, Brown AR, Loutet SA, Flannagan RS, Campopiano DJ, et al. A putative gene cluster for aminoarabinose biosynthesis is essential for Burkholderia cenocepacia viability. J Bacteriol. 2007;189:3639-44.

74. Kashima T, Nakamura T, Tojo S. Uric acid recycling in the shield bug, Parastrachia japonensis (Hemiptera: Parastrachiidae), during diapause. J Insect Physiol. 2006;52:816-25.

75. Zientz E, Dandekar T, Gross R. Metabolic interdependence of obligate intracellular bacteria and their insect hosts. Microbiol Mol Biol Rev. 2004;68:745-70.

76. Patino-Navarrete R, Moya A, Latorre A, Pereto J. Comparative genomics of Blattabacterium cuenoti: the frozen legacy of an ancient endosymbiont genome. Genome Biol Evol. 2013;5:35161.

77. Gil R, Silva FJ, Zientz E, Delmotte F, González-Candelas F, Latorre A, et al. The genome sequence of Blochmannia 
floridanus: comparative analysis of reduced genomes. Proc Natl Acad Sci USA. 2003;100:9388-93.

78. Shigenobu S, Watanabe H, Hattori M, Sakaki Y, Ishikawa H. Genome sequence of the endocellular bacterial symbiont of aphids Buchnera sp. APS. Nature. 2000;407:81-86.

79. Santos-Garcia D, Latorre A, Moya A, Gibbs G, Hartung V, Dettner $\mathrm{K}$, et al. Small but powerful, the primary endosymbiont of moss bugs, Candidatus Evansia muelleri, holds a reduced genome with large biosynthetic capabilities. Genome Biol Evol. 2014;6:1875-93.

80. Douglas AE. Nutritional interactions in insect-microbial symbioses: aphids and their symbiotic bacteria Buchnera. Annu Rev Entomol. 1998;43:17-37.

81. Engel P, Moran NA. The gut microbiota of insects-diversity in structure and function. FEMS Microbiol Rev. 2013;37:699-735.

82. Salem H, Bauer E, Strauss AS, Vogel H, Marz M, Kaltenpoth M. Vitamin supplementation by gut symbionts ensures metabolic homeostasis in an insect host. Proc R Soc Lond B Biol Sci. 2014;281:20141838.

83. Sharma S, Kaur M, Goyal R, Gill BS. Physical characteristics and nutritional composition of some new soybean (Glycine max (L.) Merrill) genotypes. J Food Sci Technol. 2014;51:551-7.

84. Edelman M, Colt M. Nutrient value of leaf vs. seed. Front Chem. 2016;4:32.

85. Mehdiabadi NJ, Schultz TR. Natural history and phylogeny of the fungus-farming ants (Hymenoptera: Formicidae: Myrmicinae: Attini). Myrmecol News. 2010;13:37-55.

86. Torrallardona D, Harris CI, Fuller MF. Microbial amino acid synthesis and utilization in rats: the role of coprophagy. Br J Nutr. 1996;76:701-9.

87. Sakaguchi E. Digestive strategies of small hindgut fermenters. Anim Sci J. 2003;74:327-37. 